\title{
Behavioural interventions targeting physical activity and diet behaviours in pediatric oncology: A Scoping Review
}

\author{
Catherine Demers ${ }^{1}$, Annie Brochu ${ }^{2}$, Johanne Higgins ${ }^{3}$, and Isabelle Gélinas ${ }^{1}$ \\ ${ }^{1}$ McGill University \\ ${ }^{2}$ CHU Ste-Justine \\ ${ }^{3}$ Université de Montréal Faculté de Médecine
}

July 20, 2020

\begin{abstract}
As the effects of cancer and its treatment have long-lasting negative impacts on the health and quality of life of survivors, there is a need to explore new avenues to optimize long-term patient outcomes in pediatric oncology. Therefore, this scoping review aims to report on the state of the evidence on the use and effects of behavioural interventions targeting physical activity and diet behaviours in pediatric oncology. Fourteen quantitative studies were included. Studies evaluated a combination of two or three different modalities, including education $(n=11)$, physical activity $(n=6)$, psychosocial support or training $(n=6)$, reward system $(n=2)$ and adventure-based activities $(n=1)$. Overall, behavioural interventions in pediatric oncology appear beneficial; however, no conclusive evidence favouring specific interventions were identified.
\end{abstract}

\section{ABSTRACT}

As the effects of cancer and its treatment have long-lasting negative impacts on the health and quality of life of survivors, there is a need to explore new avenues to optimize long-term patient outcomes in pediatric oncology. Therefore, this scoping review aims to report on the state of the evidence on the use and effects of behavioural interventions targeting physical activity and diet behaviours in pediatric oncology. Fourteen quantitative studies were included. Studies evaluated a combination of two or three different modalities, including education $(n=11)$, physical activity $(n=6)$, psychosocial support or training $(n=6)$, reward system $(\mathrm{n}=2)$ and adventure-based activities $(\mathrm{n}=1)$. Overall, behavioural interventions in pediatric oncology appear beneficial; however, no conclusive evidence favouring specific interventions were identified.

\section{INTRODUCTION}

Progress in childhood cancer treatment, diagnosis and management have resulted in childhood cancer survival rates of over $80 \%$ in North America in the past decades [1,2]. However, concurrent with this success comes an increased appreciation of the late effects resulting from the disease and its treatment that have been extensively described in the literature [3-5]. It is estimated that $62.3 \%$ of adult survivors of childhood cancer suffer from at least one chronic health condition, $27.5 \%$ have a severe or life-threatening condition [6] and $95 \%$ will have a significant health-related issue by the time they are 45 years of age [7].

For many cancer-related complications, behavioural modifications represent the primary method of risk modification available to survivors [8]. Although exercising, having a healthy diet, and adopting other healthy behaviours are beneficial for everyone, the importance of a healthy lifestyle is critical for pediatric cancer patients who are at increased risk of adverse health problems that could be potentially preventable. Lifestyle practices are well-recognized modifiable factors that contribute to lower the risk of cardiometabolic complications [9], one of the treatment-related long-term complication prevalent in this population [10, 11], 
and might increase the quality of life of survivors [12]. A review of social cognitive theory-based interventions in adult cancer survivors demonstrated promise in improving physical activity (PA) and diet behaviour [13]. Whereas interventions targeting one specific behaviour change such as exercise interventions have been the subject of reviews [14-17], there are currently no evidence-based recommendations to guide the promotion of more global behavioural intervention changes in the pediatric oncology population and little is known about complex health promotion interventions and their effectiveness.

Hence, the purpose of this scoping review is to report on the extent of what is known on the use of complex interventions or multimodal programs addressing PA and diet behaviour for children with cancer or childhood cancer survivors (CCS) and their reported findings. More specifically, the aims of this study are to examine the extent, range and nature of (1) the study populations, (2) the interventions or programs, and (3) the findings regarding the effects on health behaviours, patient outcomes, and clinical recommendations.

\section{METHODS}

The scoping review was conducted following the methodological framework by Arksey \& O'Malley [18], with improved recommendations by Levac et al. [19] to examine and summarize the extent, range, and nature of complex interventions or multimodal programs targeting PA and/or nutrition as well as their findings in pediatric oncology. The adopted strategy involved searching for research evidence via electronic databases (Embase, CINAHL, Ovid MedLine, and PsychINFO), using snowballing technique of the reference lists of selected studies and hand searching of key journals. For the electronic databases search, no limit in publication dates was set and a combination of key words and MeSH terms were used based upon the identified core concepts of the research question. The search strategy for electronic databases was developed from the research question and definitions of key concepts with the help of a librarian. Materials in English and French were included.

Prior to study selection, inclusion and exclusion criteria were created. Then, two reviewers (CD and AB) independently screened the title and abstract of studies for inclusion. Disagreements were resolved by consensus. Studies were included for full-text review if they (i) included children with cancer or survivors of childhood cancer that were diagnosed before the age of 21 , (ii) consisted of complex interventions or multimodal program, (iii) interventions targeted PA and/or nutrition. Behavioural complex interventions or programs were defined as broad interventions that are built from several interacting components that aim to change health behaviours and improve patient outcomes. The second level of screening involved reading the full text of each article, which was done by CD. AB was consulted as needed for further clarification of any ambiguities. Full-text review included all methodologies and excluded syntheses or reviews of existing evidence, theoretical and empirical articles, conference abstracts, and editorials. Multiple articles that provided results from the same study were grouped together for analyses. For intervention studies that had been preceded by published pilot studies, only the more recent version was discussed. A charting form was developed to retrieve the following data from the selected studies: authors, year of publication, study design, study population, sample size, intervention/program description, and findings (see Table 1).

In accordance with Levac et al. [19] and Colquhoun et al. [20] guidelines, we conducted a descriptive numerical and a thematic analysis. The descriptive numerical analysis focused on the characteristics of the studies (e.g. age of patients, program duration). We conducted a thematic analysis from the extracted data to answer our scoping review questions. Themes emerged around: (i) population addressed, (ii) type of interventions, (iii) patient outcomes, and (iv) clinical recommendations.

\section{RESULTS}

\section{Descriptive numerical analysis}

The initial search included all studies published before December 2019, which is when the online searches were executed. As outlined in Fig. 1, a total of 831 articles were initially retrieved through the database search. Six additional studies were identified through cross-references and hand searching. Of those, 32 articles were retrieved for full-text review and 14 were eventually retained for the final analysis. The two 
study designs employed were randomized control trials (RCT, 71\%) and quasi-experimental designs with pre-/post-study assessments (29\%). A summary if the studies included in this review is presented in Table 1.

Number of participants

Studies included a total of 1000 participants, ranging from 10 [21] to 267 participants [22].

\section{Age of participants}

The age of the participants ranged from three to 34 years old. Four studies targeted adolescents and/or young adults (11-34 years old) [21-24], seven included children and adolescents (4-20 years old) [25-31] and three were designed for the caregivers of younger children (3-13 years old) [32-34]. No studies included families of children younger than 3 years of age.

\section{Program duration}

The shorter program proposed a half-day intervention, with the post-intervention assessment conducted 3 months following the intervention [23], whereas the longest proposed a 2.5-year program beginning after diagnosis and continuing through the end of treatment [27]. Most interventions had a duration ranging between 6 to 12 weeks.

\section{Qualitative analysis}

\section{Population}

Most studies included cancer patients with mixed diagnoses groups, except for four studies that included specifically patients with acute lymphoblastic leukemia $[27,29,32,34]$ and one that included only children with leukemia and brain tumours [31]. Moreover, the majority of studies had few inclusion criteria, with the exception of two studies that were designed specifically for CCS with obesity, defined as a body mass index (BMI) [?] $85^{\text {th }}$ percentile [29, 33] and one for survivors who reported symptoms of fatigue and had not engaged in PA in the previous 6 months [26].

Eight studies targeted the cancer patients or survivors [21-24, 26, 28, 30, 31], five were family-oriented [25, $27,29,32,34]$ and one was designed for parents [33].

Participants in nine studies were survivors [21-24, 26, 28, 29, 31, 33], whereas the remaining five studies recruited children undergoing treatment $[25,27,30,32,34]$.

Nine studies were conducted in the USA [22-24, 27, 29, 31-34], two in Hong Kong [26, 30], one in Canada [21], one in the Netherlands [25] and one in Taiwan[28].

\section{Interventions or Programs}

The more frequent type of health behaviours addressed in each individual study were PA and nutrition, in 13 and seven studies respectively (see Table 2). Other health behaviours studied included smoking $(\mathrm{n}=2)$ [22, 24], alcohol consumption $(n=1)$ [24], sun protection $(n=1)$ [22], health accountability $(n=1)$ [28] and self-examination $(\mathrm{n}=1)$ [22]. More than half of the studies focused on one specific health behaviour, whereas the others addressed multiple health behaviours. Furthermore, studies evaluated a combination of modalities including educational interventions $(\mathrm{n}=11)$ [21-24, 26, 28-30, 32-34], individualized or group PA interventions $(\mathrm{n}=6)[21,25,27,30-32]$, counselling $(\mathrm{n}=5)$, psychosocial support or training $(\mathrm{n}=6)[22,23,25,27,33,34]$, reward system (i.e. healthy goods and services) $(\mathrm{n}=2)[24,31]$ and adventure-based activities $(\mathrm{n}=1)$ [26]. Programs included between 2 and 3 different modalities.

Five studies were conducted in a hospital or clinic [22, 23, 25, 27, 28], four were delivered using various technologies (i.e. emails, text messages, online platforms) or telephone [24, 29, 33, 34], two were home-based $[30,32]$ and three were community-based $[21,26,31]$. 
The majority of programs or interventions were developed using a theoretical framework, the most popular being Bandura's social cognitive theory [35] used in seven studies [23, 28-31, 33, 34]. Only three studies did not report any theoretical framework $[25,27,32]$ and four integrated elements from multiple frameworks to develop their interventions $[23,26,33,34]$.

\section{Outcomes}

Following a method previously used to assess parental involvement on intervention results [36, 37], outcomes were categorized by positive or mixed results. Positive results indicated that changes occurred in the desired directions and mixed results indicated that there were positive changes, but only among one subgroup or only for some of the outcomes measured. For the purpose of this review, a third category named "no effect" was also added to indicate that there were no positive changes for any of the outcomes under study.

Seven studies included both health behaviour assessments and patient outcomes [26, 29, 30, 32-34]; whereas four focused on health behaviours $[22,24,28]$ and three on patient outcomes only [25, 27, 31]. Health behaviour assessments included, but were not limited to, PA levels, dietary recalls, health behaviour selfefficacy and consumption of alcohol and drinking for adult survivors. The most frequent patient outcomes were body mass index (BMI), physical fitness and quality of life. Most studies reported mixed results, while one study reported positive findings across all patient outcomes or health behaviours assessments [30] and two not conclusive results $[24,27]$.

Nine studies also reported on feasibility outcomes, such as retention rate, acceptability, and participants' satisfaction. All of them reported positive findings, except for one that reported mixed findings [31], supporting feasibility and acceptability of their respective interventions or program that were safely and successfully implemented in the childhood cancer population.

Ten studies conducted the assessments at short-term; either directly at the end of the intervention [27-29, 32] [31] or between 1 and 4 months post-intervention [23, 24, 28, 29, 33]. The 4 remaining studies conducted long-term assessments, i.e. 12 months after the program or interventions $[21,22,25,26]$.

\section{Clinical implications}

To increase the effectiveness of interventions, various recommendations were made by the authors to guide either clinical practice or future studies (see Table 3). Some recommendations were made regarding important elements that should be included or considered, including supporting participants' self-efficacy, which is defined as an individual's self-judgment of their own abilities to execute certain behaviours that determine the likelihood of a person engaging in or avoiding certain behaviours [38]. Indeed, interventions that address self-efficacy elements may increase children or survivors' confidence in their abilities to initiate and maintain healthy behaviours [28]. For example, it was found that with increased self-efficacy, children with cancer are more likely to adopt and maintain regular PA [30]. Also, psychological variables are deemed important intervention targets to improve quality of life [25]. For example, cognitive deficits such as information processing problems should be addressed [29].

The engagement of families, especially parents is particularly important [34]. Indeed, caregivers serving as role models of healthy eating and exercise facilitate family changes [33]. Positive social interactions and encouragement with the mentor was found to be associated with greater adherence to healthy behaviours in one of the studies; the authors therefore recommended enlisting the support of parents or friends to provide additional social support to the CCS [31]. Furthermore, authors support more frequent contact with the interventionists as participants seem to need the continuing support on the lifelong commitment to healthy behaviours to moderate the late effects of therapy [27].

Another popular recommendation was to use targeted, individualized programs and age-appropriate approaches, as they might be better than standard or generic programs to increase the effects of the program $[21,23,25,32]$. Making the messages specific to the needs of young cancer survivors would likely enhance their relevance as well as increase the engagement and satisfaction of survivors with the content [24]. 
Multimodal approaches have contributed to compliance and overall success in reaching targeted outcomes [29]. Indeed, multimodal seemed preferable to education only, as provision of information related to adverse outcomes is necessary, but was found to be not sufficient to produce significant changes in participants' health outcomes [22]. Furthermore, the use of technology offers a feasible, relatively low-cost alternative to more in-person intensive interventions in this at-risk but sparse population because it can be distributed across time and geography; however, personal contact also appeared to help compliance with protocol and follow-up [29].

Finally, regarding the point over the course of treatment and survivorship that interventions might be best implemented, Cox et al. [27] concluded that it may not be feasible during early treatment owing to the child's responses to the disease and treatment. Indeed, shortly after the diagnosis and following the participants through the end of therapy may have resulted in the study participants and/or parents being too sick and/or overwhelmed to complete the intervention with the frequency and intensity necessary to improve function. They suggested offering study participation at maintenance, in contrast to within 10 days after diagnosis, so that parents and children will have had a considerable period of time to adjust to the diagnosis and to have hurdled the worst treatment and disease effects. Fear, anxiety, symptoms, and overall well-being will no longer be the significant threats that they were at diagnosis, thereby potentially facilitating greater participation and commitment to the intervention. Another early lifestyle intervention concluded that it was feasible, but that the results did not show significant changes in the targeted outcomes [34]. For survivors, it was found that trying to recruit after treatment was difficult as families are often trying to forget their cancer and hospital experiences and, similarly, too long after (e.g. more than 3 years) was also difficult as families are likely to have created a new normal. The average time since treatment ended for participants recruited in their study was 1.96 years [33].

\section{DISCUSSION}

The present scoping review sheds light on the dominant areas of research in terms of target population, intervention type, and outcomes for complex interventions or multimodal programs targeting healthy behaviours in children with cancer. Due to a paucity of studies, and the heterogeneity of the studies included in this review, identifying the best health promotion interventions or program is challenging. The interventions typically focused on PA alone or in combination with other health behaviours, the majority had a short duration ( $<6$ months), and few conducted long-term assessments. Furthermore, no studies included families of children younger than three years of age and few recruited patients who were still undergoing treatment. There are a lot of challenges that can be encountered in developing and administering interventions with children who are severely ill, which could explain why so little intervention effort has been made.

The few studies published demonstrate that it is feasible to implement these types of interventions or programs and that it can potentially improve patient outcomes or healthy behaviours practises. Furthermore, popular recommendations include the individualization of interventions, the use multiple modalities and the engagement of families. The results of this study are consistent with other reviews; one concluding that diet and exercise interventions are feasible [39] and another one that lifestyle technology-based interventions demonstrated high feasibility and acceptability rates in the pediatric oncology and survivors of childhood cancer populations [40]. Moreover, a recent review of health behaviour in CCS interventions confirmed a gap in interventions designed for younger cancer survivors ( $<8$ years of age) and that engaged parents, who are critical to child behaviour change [41].

A crucial aspect in the evaluation of complex behavioural interventions is the choice of outcome measures. Precise and reliable measures of both behaviours targeted by the intervention and the health outcomes should be included for a comprehensive evaluation of behavioural interventions. Esbenshade and Ness [39] have suggested to pick out outcomes that are reliable and reproducible as well as to standardize outcome measures so that studies can be compared and combined. Furthermore, long-term follow-up may be needed to determine whether the downstream effects on the health outcome predicted by the change in behaviour occurred or whether the short-term changes, such as the change in behaviour, persisted [42]. Thus, implementing programs of longer duration should be encouraged. Collecting data over an extended period of time 
(e.g. more than 12 months after starting the intervention) would allow clinicians or researchers to evaluate the long-term effects and benefits of the interventions or program on outcomes such as quality of life, which requires an extended period to respond the intervention compared with other outcomes, for example levels of PA.

Consistent with the recommendations from Stern [33] and Zhang [34] to engage families in behavioural interventions, a systematic review that examined parental involvement in diet and PA studies in CCS concluded that adding a parental component may improve health promotion interventions for CCS [36]. Indeed, in their review, studies with no or indirect parental involvement had lower amounts of success than studies with direct parental involvement. Furthermore, as some research has shown that parents remain involved in the healthcare of survivors even into adulthood [43], parental involvement should be considered throughout the continuum of care and regardless of the survivors' age. Parents of CCS are also known to experience psychosocial issues related to the child's cancer and treatment that can affect their family life [44], such as being overprotective and restricting children from participating in physical activities [45] or adopting a parenting style that is associated with increased junk food consumption [46]. Therefore, addressing parenting practises and offering psychosocial support or training to families may be beneficial for both parents and children with cancer.

According to Short et al. [47], programs addressing the promotion of health behaviours such as PA should be theory-based in order to maximise effectiveness. Indeed, there is a growing recognition that theorybased behaviour change interventions are more effective than approaches that do not rely on theory [48]. Among other reasons, theory-based interventions can help pinpoint what factors need to be considered before developing a program or intervention, it can provide insight into how to shape strategies to address these factors, and can provide information on how or why the intervention works. The theoretical framework that has been used the most in the field of pediatric oncology is the Social Cognitive Theory (SCT) [49], which is a theory that has shown particular promise also in the field of PA promotion for breast cancer survivors [47]. However, the application of SCT or any other theoretical framework to promote healthy behaviours in oncology is still in its infancy and further research is needed to optimize their use.

Interventions to change health behaviours such as adopting a healthy diet and practicing frequent PA have huge potential to make a positive impact on both the individual themselves and the overburdened healthcare system [50]. Furthermore, there is growing evidence that multiple-behaviour interventions promise to have much greater impact on public health than single-behaviour interventions [51]. The key idea that underpins behavioural change is to deliver interventions comprised of various change-generating techniques in order to promote a change in behaviour, which will subsequently have an effect on health outcomes. The same rigorous experimental methods that have characterized drug-based clinical treatments should be applied to those studies, which is unfortunately not always the case. Indeed, methods must be devised to measure what was previously thought to be unmeasurable and assessments conducted in a reproducible and valid fashion, even for the measures of subjective states [52].

Limitations from this review include the fact that this is a developing field of research, with the oldest article published in 1999, with few studies addressing the research question. The high variability in intervention type and outcome measures across studies made comparison of results difficult and meant we were not able to identify attributes of studies that were effective. Furthermore, many of the included studies had small sample sizes, biases and short follow-up duration. This review underlines the need for further well-designed trials using standardized outcome measures to be implemented in this population as well as the gaps in the evidence base.

\section{CONCLUSION}

This review highlights the lack of studies on interventions to optimise long-term health in pediatric oncology, especially for younger children and patients still undergoing cancer treatment. No conclusive evidence favouring specific interventions were identified, although there is preliminary evidence that health promotion interventions are feasible and potentially beneficial for children with cancer and survivors. These results 
also provide insights that are useful in integrating future health promotion interventions in childhood cancer patients and survivors. Future research is vital in identifying and defining the most efficient methods to implement these programs and optimize patient outcomes.

\section{CONFLICT OF INTEREST STATEMENT}

All authors declare no conflict of interest.

\section{ACKNOWLEDGEMENT}

This research was supported by the Fondation des Gouverneurs de l'Espoir.

\section{REFERENCES}

1. Ontario, P.O.G.o., Childhood Cancer in Ontario 1986 - 2015: A Surveillance Report from the Pediatric Oncology Group of Ontario . 2018, Pediatric Oncology Group of Ontario: Toronto.

2. Society, A.C., Facts \& Figures 2018 . 2018, American Cancer Society: Atlanta, GA.

3. Galligan, A.J., Childhood Cancer Survivorship and Long-Term Outcomes. Adv Pediatr, 2017. 64 (1): p. 133-169.

4. Landier, W., S. Armenian, and S. Bhatia, Late effects of childhood cancer and its treatment. Pediatr Clin North Am, 2015.62 (1): p. 275-300.

5. Dickerman, J.D., The late effects of childhood cancer therapy.Pediatrics, 2007. 119 (3): p. 554-68.

6. Oeffinger, K.C., et al., Chronic health conditions in adult survivors of childhood cancer. N Engl J Med, 2006. 355 (15): p. 1572-82.

7. Hudson, M.M., et al., Clinical ascertainment of health outcomes among adults treated for childhood cancer. JAMA, 2013.309 (22): p. 2371-2381.

8. Demark-Wahnefried, W. and L.W. Jones, Promoting a healthy lifestyle among cancer survivors. Hematol Oncol Clin North Am, 2008.22 (2): p. 319-42, viii.

9. Leiter, L.A., et al., Cardiometabolic risk in Canada: a detailed analysis and position paper by the cardiometabolic risk working group. Can J Cardiol, 2011. 27 (2): p. e1-e33.

10. Mertens, A.C., et al., Late mortality experience in five-year survivors of childhood and adolescent cancer: the Childhood Cancer Survivor Study. J Clin Oncol, 2001. 19 (13): p. 3163-3172.

11. Nottage, K.A., et al., Metabolic syndrome and cardiovascular risk among long-term survivors of acute lymphoblastic leukaemia - From the St. Jude Lifetime Cohort. Br J Haematol, 2014. 165 (3): p. 364-74.

12. van Brussel, M., et al., Is physical fitness decreased in survivors of childhood leukemia? A systematic review. . Leukemia, 2005.19 (1): p. 13-7.

13. Stacey, F.G., et al., A systematic review and meta-analysis of social cognitive theory-based physical activity and/or nutrition behavior change interventions for cancer survivors. J Cancer Surviv, 2015. 9 (2): p. $305-38$.

14. Baumann, F.T., W. Bloch, and J. Beulertz, Clinical exercise interventions in pediatric oncology: a systematic review. Pediatr Res, 2013. 74 (4): p. 366-74.

15. Braam, K.I., et al., Physical exercise training interventions for children and young adults during and after treatment for childhood cancer. Cochrane Database Syst Rev, 2016. 3 : p. CD008796.

16. Huang, T.T. and K.K. Ness, Exercise interventions in children with cancer: a review. Int J Pediatr, 2011. 2011 : p. 461512. 
17. Zhang, F.F., M.J. Kelly, and A. Must, Early Nutrition and Physical Activity Interventions in Childhood Cancer Survivors. Curr Obes Rep, 2017. 6 (2): p. 168-177.

18. Arksey, H. and L. O'Malley, Scoping studies: towards a methodological framework Int J Soc Res Methodol, 2005. 8 : p. 19-32.

19. Levac, D., H. Colquhoun, and K.K. O'Brien, Scoping studies: advancing the methodology. Implement Sci, 2010. 5 : p. 69.

20. Colquhoun, H.L., et al., Scoping reviews: time for clarity in definition, methods, and reporting. J Clin Epidemiol, 2014.67 (12): p. 1291-4.

21. Keats, M.R. and S.N. Culos-Reed, A community-based physical activity program for adolescents with cancer (project TREK): program feasibility and preliminary findings. J Pediatr Hematol Oncol, 2008.30 (4): p. $272-80$.

22. Hudson, M.M., et al., Multi-component behavioral intervention to promote health protective behaviors in childhood cancer survivors: the protect study. Med Pediatr Oncol, 2002. 39 (1): p. 2-1; discussion 2.

23. Mays, D., et al., Efficacy of the Survivor Health and Resilience Education (SHARE) program to improve bone health behaviors among adolescent survivors of childhood cancer. Ann Behav Med, 2011.42 (1): p. 91-8.

24. Berg, C.J., et al., Pilot results of an online intervention targeting health promoting behaviors among young adult cancer survivors. Psychooncology, 2014. 23 (10): p. 1196-9.

25. Braam, K.I., et al., Effects of a combined physical and psychosocial training for children with cancer: a randomized controlled trial. BMC Cancer, 2018. 18 (1): p. 1289.

26. Li, W.H.C., et al., Adventure-based training to promote physical activity and reduce fatigue among childhood cancer survivors: A randomized controlled trial. Int J Nurs Stud, 2018. 83 : p. 65-74.

27. Cox, C.L., et al., Modifying bone mineral density, physical function, and quality of life in children with acute lymphoblastic leukemia. Pediatr Blood Cancer, 2018. 65 (4).

28. Wu, L.M., et al., Tailored education enhances healthy behaviour self-efficacy in childhood cancer survivors: A randomised controlled study with a 4-month follow-up. Eur J Cancer Care (Engl), 2019. 28 (4): p. e13063.

29. Huang, J.S., et al., Fit4Life: a weight loss intervention for children who have survived childhood leukemia. Pediatr Blood Cancer, 2014. 61 (5): p. 894-900.

30. Lam, K.K.W., et al., An integrated experiential training programme with coaching to promote physical activity, and reduce fatigue among children with cancer: A randomised controlled trial. Patient Educ Couns, 2018. 101 (11): p. 1947-1956.

31. Gilliam, M.B., et al., A Pilot Study Evaluation of a Web-Based Token Economy to Increase Adherence with a Community-Based Exercise Intervention in Child and Adolescent Cancer Survivors. Rehab Oncol, 2011. 29 (2): p. 16-22.

32. Moyer-Mileur, L.J., L. Ransdell, and C.S. Bruggers, Fitness of children with standard-risk acute lymphoblastic leukemia during maintenance therapy: response to a home-based exercise and nutrition program. J Pediatr Hematol Oncol, 2009. 31 (4): p. 259-66.

33. Stern, M., et al., NOURISH-T: Targeting caregivers to improve health behaviors in pediatric cancer survivors with obesity. Pediatr Blood Cancer, 2018. 65 (5): p. e26941.

34. Zhang, F.F., et al., Early Lifestyle Intervention for Obesity Prevention in Pediatric Survivors of Acute Lymphoblastic Leukemia.Nutrients, 2019. 11 (11).

35. Bandura, A., Self-efficacy: toward a unifying theory of behavioral change. Psychol Rev, 1977. 84 (2): p. 191-215. 
36. Raber, M., et al., Parental involvement in exercise and diet interventions for childhood cancer survivors: a systematic review.Pediatr Res, 2016. 80 (3): p. 338-46.

37. Hingle, M.D., et al., Parental involvement in interventions to improve child dietary intake: a systematic review. Prev Med, 2010.51 (2): p. 103-11.

38. Bandura, A., Self-Efficacy: The Exercise of Control . 1997, New York: Freeman.

39. Esbenshade, A.J. and K.K. Ness, Dietary and Exercise Interventions for Pediatric Oncology Patients: The Way Forward. J Natl Cancer Inst Monogr, 2019. 2019 (54): p. 157-162.

40. Kopp, L.M., et al., Lifestyle behavior interventions delivered using technology in childhood, adolescent, and young adult cancer survivors: A systematic review. Pediatr Blood Cancer, 2017.64 (1): p. 13-17.

41. Brier, M.J., L.A. Schwartz, and A.E. Kazak, Psychosocial, health-promotion, and neurocognitive interventions for survivors of childhood cancer: a systematic review. Health Psychol, 2015.34 (2): p. 130-48.

42. Craig, P., et al., Developing and evaluating complex interventions: the new Medical Research Council guidance. BMJ, 2008.337 : p. a1655.

43. Kinahan, K.E., et al., Adult survivors of childhood cancer and their parents: experiences with survivorship and long-term follow-up. J Pediatr Hematol Oncol, 2008. 30 (9): p. 651-8.

44. Long, K.A. and A.L. Marsland, Family adjustment to childhood cancer: a systematic review. Clin Child Fam Psychol Rev, 2011.14 (1): p. 57-88.

45. Wakefield, C.E., et al., Parental adjustment to the completion of their child's cancer treatment. Pediatr Blood Cancer, 2011.56 (4): p. 524-31.

46. Williams, L.K., K.E. Lamb, and M.C. McCarthy, Parenting Behaviors and Nutrition in Children with Leukemia. J Clin Psychol Med Settings, 2015. 22 (4): p. 279-90.

47. Short, C.E., E.L. James, and R.C. Plotnikoff, How social cognitive theory can help oncology-based health professionals promote physical activity among breast cancer survivors. Eur J Oncol Nurs, 2013. 17 (4): p. $482-9$.

48. Glanz, K. and D.B. Bishop, The role of behavioral science theory in development and implementation of public health interventions. Annu Rev Public Health, 2010. 31 : p. 399-418.

49. Bandura, A., Health promotion by social cognitive means.Health Educ Behav, 2004. 31 (2): p. 143-64.

50. Toorney, E., Opening the «black box $\gg$ : the need for fidelity and process evaluation of behaviour change interventions . 2017.

51. Nigg, C.R., J.P. Allegrante, and M. Ory, Theory-comparison and multiple-behavior research: common themes advancing health behavior research. Health Educ Res, 2002.17 (5): p. 670-9.

52. Streiner, D.L. and G.R. Norman, Health measurement scales: A practical guide to their development and use . $4^{\text {th }}$ Edition ed. 2008, Oxford: Oxford University Press.

FIGURE LEGEND

$\overline{\text { Figure } 1}$ Flow diagram of the scoping review process

\section{Hosted file}

Table 1.docx available at https://authorea.com/users/343717/articles/470329-behaviouralinterventions-targeting-physical-activity-and-diet-behaviours-in-pediatric-oncology-ascoping-review 


\section{Hosted file}

Table 2.docx available at https://authorea.com/users/343717/articles/470329-behaviouralinterventions-targeting-physical-activity-and-diet-behaviours-in-pediatric-oncology-ascoping-review

\section{Hosted file}

Table 3.docx available at https://authorea.com/users/343717/articles/470329-behaviouralinterventions-targeting-physical-activity-and-diet-behaviours-in-pediatric-oncology-ascoping-review

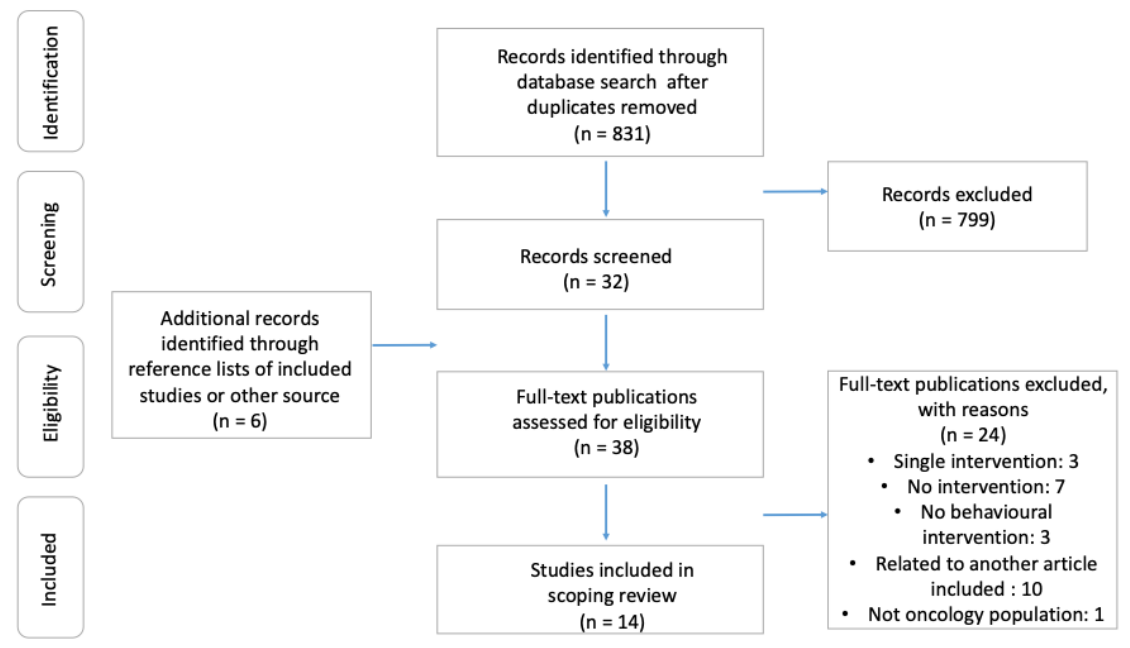

